OPEN ACCESS

Edited by:

Zhenyu Jia,

University of California, Riverside,

United States

Reviewed by:

Tolga Turan

AbbVie, United States

Shukui Wang,

Nanjing Medical University, China

*Correspondence:

Ren-Xiong Wei

w/s0821@126.com

Specialty section:

This article was submitted to Molecular and Cellular Oncology,

a section of the journal

Frontiers in Oncology

Received: 07 May 2020

Accepted: 24 June 2020

Published: 24 July 2020

Citation:

Gu $H-Y$, Lin L-L, Zhang $C$, Yang $M$,

Zhong H-C and Wei R-X (2020) The

Potential of Five Immune-Related

Prognostic Genes to Predict Survival and Response to Immune Checkpoint Inhibitors for Soft Tissue Sarcomas

Based on Multi-Omic Study.

Front. Oncol. 10:1317.

doi: 10.3389/fonc.2020.01317

\section{The Potential of Five} Immune-Related Prognostic Genes to Predict Survival and Response to Immune Checkpoint Inhibitors for Soft Tissue Sarcomas Based on Multi-Omic Study

\author{
Hui-Yun Gu ${ }^{1}$, Lu-Lu Lin ${ }^{2}$, Chao Zhang ${ }^{3,4}$, Min Yang ${ }^{1}$, Hou-Cheng Zhong ${ }^{1}$ and \\ Ren-Xiong Wei ${ }^{1 *}$
}

${ }^{1}$ Department of Spine and Orthopedic Oncology, Zhongnan Hospital of Wuhan University, Wuhan, China, ${ }^{2}$ Department of Pathology and Pathophysiology, School of Basic Medicine, Wuhan University, Wuhan, China, ${ }^{3}$ Center for Evidence-Based Medicine and Clinical Research, Taihe Hospital, Hubei University of Medicine, Shiyan, China, ${ }^{4}$ Department of Oncology, Taihe Hospital, Hubei University of Medicine, Shiyan, China

Low response rates to immunotherapy have been reported in soft tissue sarcoma (STS). There are few predictive biomarkers of response, and the tumor immune microenvironment associated with progression and prognosis remains unclear in STS. Gene expression data from the Cancer Genome Atlas were used to identify the immune-related prognostic genes (IRPGs) and construct the immune gene-related prognostic model (IGRPM). The tumor immune microenvironment was characterized to reveal differences between patients with different prognoses. Furthermore, somatic mutation data and DNA methylation data were analyzed to understand the underlying mechanism leading to different prognoses. The IGRPM was constructed using five IRPGs (IFIH1, CTSG, STC2, SECTM1, and BIRC5). Two groups (high- and low-risk patients) were identified based on the risk score. Low-risk patients with higher overall survival time had higher immune scores, more immune cell infiltration (e.g., CD8 T cell and activated natural killer cells), higher expression of immune-stimulating molecules, higher stimulating cytokines and corresponding receptors, higher innate immunity molecules, and stronger antigen-presenting capacity. However, inhibition of immunity was observed in low-risk patients owing to the higher expression of immune checkpoint molecules and inhibiting cytokines. High-risk patients had high tumor mutation burden, which did not significantly influence survival. Gene set enrichment analysis further revealed that pathways of cell cycle and cancers were activated in high-risk patients. DNA methylation analysis indicated that relative high methylation was associated with better overall survival. Finally, the age, mitotic counts, and risk scores were independent prognostic factors for STS. Five IRPGs performed well in risk stratification of patients and are candidate biomarkers for predicting response to immunotherapy. Differences observed through the multi-omic 
study of patients with different prognoses may reveal the underlying mechanism of the development and progression of STS, and thereby improve treatment.

Keywords: immune-related prognostic genes (model), tumor immune microenvironment, somatic variants, DNA methylation, multi-omic study

\section{INTRODUCTION}

Unlike cancers with epithelial origins, soft tissue sarcoma (STS) evolved from mesenchymal tissues in different anatomical sites (1). Despite its lower incidence vs. cancers, STS with high aggressive behavior was responsible for 5,270 deaths according to the 2019 Cancer Statistics (2). Occurrence of STS in the limbs increases the risk of disability in patients (3). Moreover, STS is characterized by high rates of relapse (4). Therefore, the treatment of STS is a challenge to most clinicians.

Patients with early-stage and localized STS $(5,6)$ can recover from radical surgical resection and achieve higher survival rates. However, patients with metastatic and recurrent STS are linked to rapid progression of disease and death due to poor response to surgical techniques and adjuvant radiotherapy (7). Conventional treatment does not meet the requirements for longer survival time and higher quality of life. An increasing number of studies revealed that the tumor microenvironment and the expression of immune checkpoint molecules accelerated the progression of cancers (8-10). The use of immune checkpoint inhibitors markedly improved the prognosis of cancers (e.g., melanoma) $(11,12)$. Based on the immune-related pathogenesis in cancers, the use of immunotherapy may promote survival in STS. Recent clinical cases reported favorable response to immune checkpoint inhibitors in classic Kaposi sarcoma (13) and myxoid chondrosarcoma (14). Nevertheless, there is insufficient evidence regarding the efficacy of immunotherapy in STS. Therefore, studies investigating the immune microenvironment or immune gene-related prognostic biomarkers, which have been identified in cervical cancer (15), lung adenocarcinoma (16), and cancers of the digestive system (17) are warranted. Such studies will assist in understanding the effect of immune infiltration on STS and predict response to immunotherapy, thereby improving efficacy against STS.

The aim of the present study was to identify immune generelated prognostic biomarkers and construct a prognostic model to determine patients with better response to immunotherapy for precision treatment in STS. Moreover, integrated analysis of multi-omic data in patients with different prognoses may elucidate the mechanism involved in tumorigenesis, metastasis, and high aggressive behavior of STS.

\section{MATERIALS AND METHODS}

\section{Collection and Preprocessing of Gene Expression and Clinical Data}

The latest version of normalized gene expression data (0720-2019) in the Cancer Genome Atlas (TCGA) database were downloaded from the UCSC (University of California, Santa Cruz) Xena browser (https://gdc.xenahubs.net). Raw gene expression data (GSE21050) (18) were also downloaded from the Gene Expression Omnibus (GEO) database. Subsequently, the gene expression profiles were preprocessed. The "RMA" algorithm (19) was used to process the GSE21050. During the procedure of probe mapping to gene symbols, mean values were maintained when multiple probes shared the same gene symbol. The gene symbols with mean expression value in all samples $<0.5$ were removed (20). For the subsequent analyses, we selected common genes with top $25 \%$ variances in TCGA and GEO datasets (21). In addition, clinical data of the TCGA and GEO samples were downloaded and preprocessed.

\section{Weighted Gene Co-expression Network Analysis (WGCNA)}

WGCNA, a new bioinformatics method, is effective in processing gene expression, proteomic, and metabolomic datasets (22, 23). It has been applied to the identification of potential crucial biomarkers in many types of diseases $(24,25)$ and key genes associated with phenotypic traits (26). There was no information regarding the survival status in the GSE21050 dataset. Therefore, WGCNA was performed to identify prognostic genes based on the expression matrix obtained from TCGA database. The gene expression matrix of genes with top $25 \%$ variance was used to construct a gene co-expression network and identify modules. Subsequently, we related modules to clinical information for the detection of modules highly associated with survival. All these procedures were performed using the "WGCNA" package (22) in R 3.5.3 software. Function enrichment analyses were conducted using the "clusterProfiler" package (27) in R software to further determine whether interesting modules were associated with survival. Following the completion of WGCNA, genes identified in the survival-related modules (interesting modules) were extracted as the preliminary immune-related prognostic genes (IRPGs) in STS.

\section{Identification and Validation of the Immune Gene-Related Prognostic Model (IGRPM)}

Immune-related genes (IRGs) identified in the Immunology Database and Analysis Portal (ImmPort) database (28) were overlapped with the prognostic genes detected in the WGCNA. The expression matrix containing common immune genes from TCGA and GEO datasets was processed through the "sva" package of R software (29) to remove the batch effect. Subsequently, it was used to identify and validate IRPGs and construct the prognostic model. Firstly, univariate Cox regression analysis of common immune genes was performed based on the "survival" package of the R software. Least absolute shrinkage and selection operator (LASSO) regression analysis (30) was applied 
for genes with $p<0.05$ in the univariate Cox regression analysis. Through the 1000 cross-validations method, more reliable IRPGs would be obtained using the "glmnet" and "survival" packages (31). Genes identified from the LASSO regression analysis were further determined via multivariate Cox regression analysis. The genes that demonstrated significance in the multivariate Cox regression analysis were considered the IRPGs in this study. According to the coefficient of IRPGs in the multivariate Cox regression analysis, the "predict" function in the "survMisc" package was used to construct the IGRPM and compute the risk score for each patient. Based on the median risk scores, patients with risk scores more than the median risk scores were classified into high-risk groups. Similarly, patients with risk scores less than the median were classified into low-risk groups. Subsequently, we plotted the receiver operating characteristic (ROC) curve using the "survivalROC" package and performed overall survival (OS) analysis to evaluate the IGRPM. Differences in gene expression, survival status, and risk scores between the high- and low-risk groups were also visualized to evaluate the prognostic model.
Finally, the GSE21050 dataset was used to validate the accuracy of the model based on the same cutoff value applied to the TCGA dataset. Owing to the lack of survival status in this dataset, the rates of metastasis-free survival were used as OS rates to validate the IGRPM.

\section{Immune Infiltration Analysis}

The immune microenvironment was investigated in this study. An algorithm using expression data for the estimation of stromal and immune cells in malignant tumors (ESTIMATE) was applied (32). The stromal score and immune score for each patient with STS were computed based on specific gene expression signatures of stromal and immune cells, and single-sample gene set enrichment analysis via the "estimate" package in the TCGA and GSE21050 datasets (32). Differences in stromal and immune cell infiltration between high- and low-risk patients were visualized via the "ggpubr" package (https://CRAN.R-project.org/package= ggpubr). Furthermore, the correlation of risk scores and immune
A
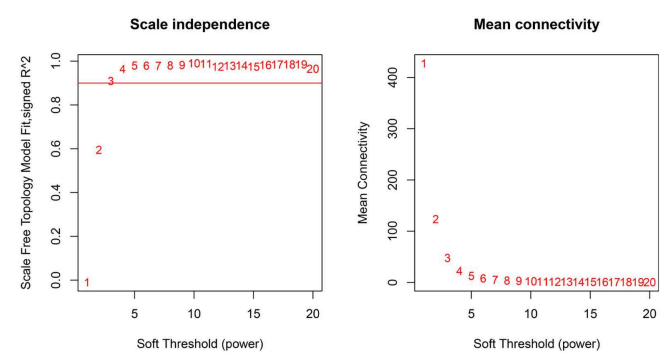

B

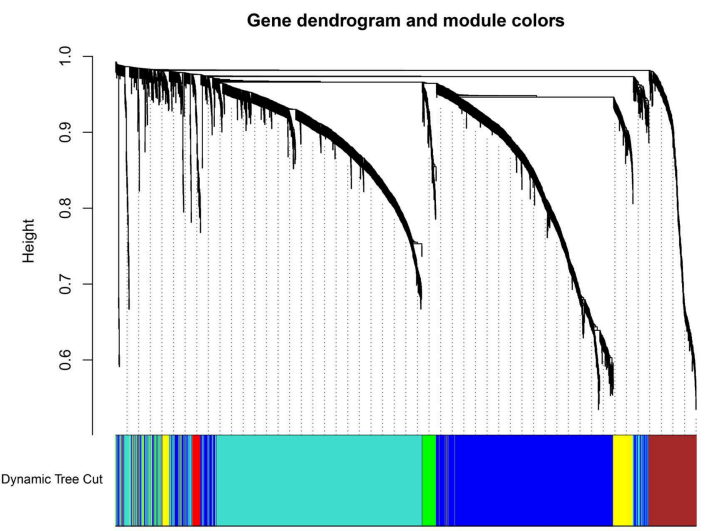

C

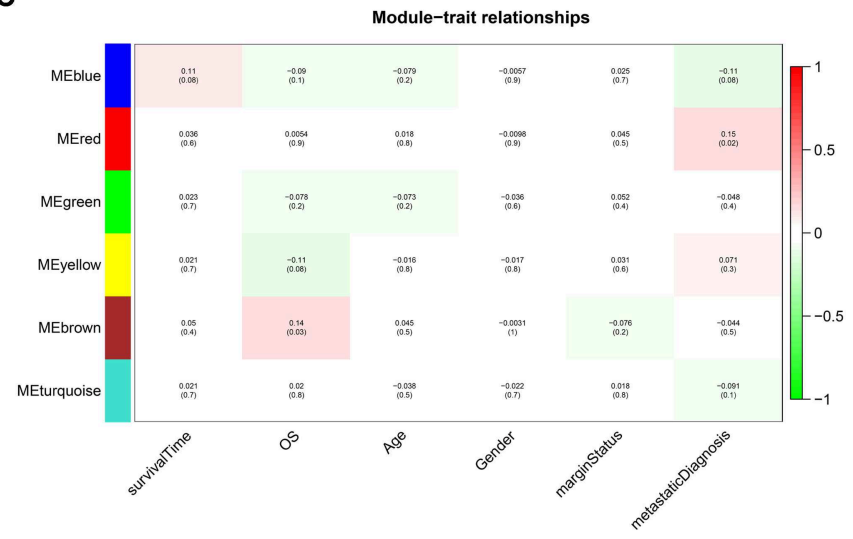

D

E
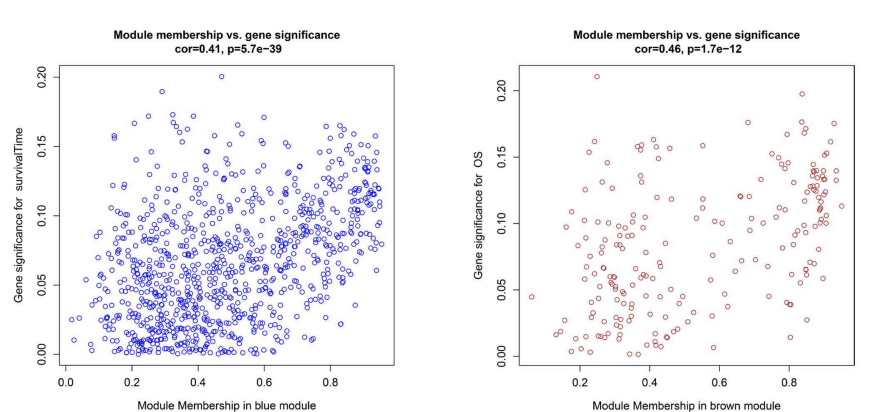

FIGURE 1 | Weighted gene co-expression network analysis (WGCNA). (A) Selection of the optimal soft threshold power, $\beta$ (optimal $\beta=3$, scale free topology index, $R^{2}=0.9$ ). (B) Module identification using the Dynamic Tree Cut method. The different color bands provide a simple visual comparison of module assignments. (C) Module-trait relationship. Six modules were identified and related to clinical traits. Each cell represented the correlation (and $p$-value) of the module with the corresponding clinical trait. OS represented the overall survival status (alive or dead). (D) Identification of genes with high significance and module membership in the survival time-related blue module. (E) Identification of genes with high significance and module membership in the OS-related brown module. 

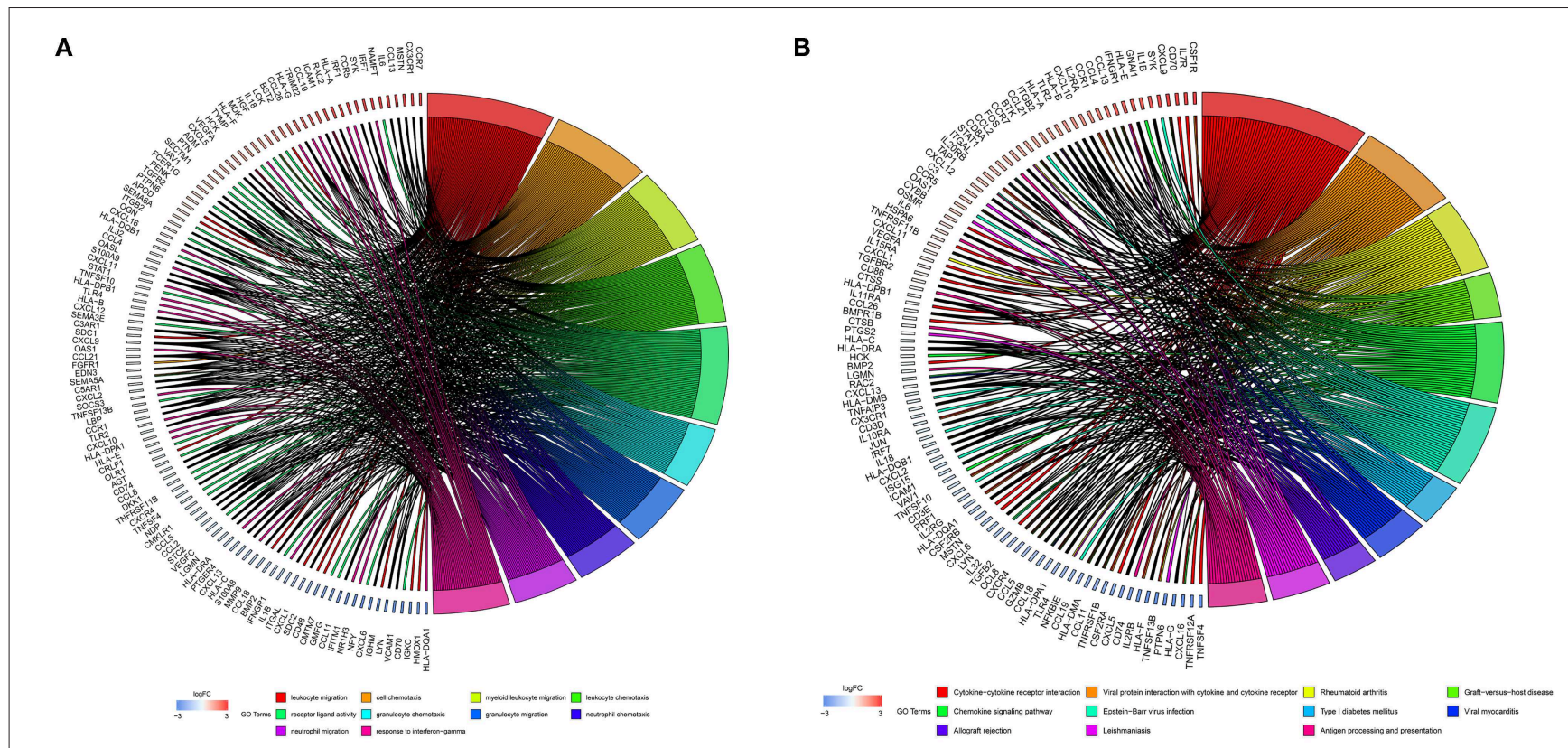

FIGURE 2 | Function enrichment analyses for the overlapped genes from the WGCNA and the Immunology Database and Analysis Portal (ImmPort) database. (A) Gene Ontology (GO) analysis for the immune genes. (B) The Kyoto Encyclopedia of Genes and Genomes (KEGG) pathways for immune genes.

scores was explored to reveal the effect of immune infiltration on prognosis. Subsequently, the CIBERSORT algorithm based on 100 permutations was used to estimate the proportions of 22 types of immune cells following the official manual provided in the CIBERSORT website (http://cibersort.stanford.edu/) (33). In addition, based on previous studies, immune-related molecules and other immune microenvironment components except immune cells (i.e., chemokines, interleukins, interferons, other cytokines, corresponding receptors of the aforementioned molecules, innate immunity molecules, immune inhibitors including common immune checkpoints, immune stimulators, and antigen-presenting molecules) were further analyzed to understand immune infiltration in STS (34-36). A $p<0.05$ denoted statistically significant difference between high- and low-risk patients.

\section{Analysis of Somatic Variants}

Notably, gene mutations may lead to neoantigen epitopes and influence the components of immune microenvironments (16). Mutation data from the VarScan2 Variant Aggregation and Masking were downloaded through the UCSC Xena website. The "maftools" package with functions for summarizing, analyzing, and visualizing mutation data was used to analyze somatic variants (37). Firstly, the overall mutation status, as well as the corresponding risk and immune scores were determined in all patients. Differential mutations were investigated in patients with different risk and immune scores to identify the crucial gene mutations associated with prognosis and immune filtration, and demonstrate the relationship between immune filtration and prognosis in STS.
Finally, the tumor mutation burden (TMB) was calculated between high- and low-risk patients based on a previous study (38).

\section{Gene Set Enrichment Analysis (GSEA)}

We performed the GSEA to elucidate the underlying mechanism involved in immune infiltration and high aggressive behavior in STS. The Kyoto Encyclopedia of Genes and Genomes (KEGG) pathway was obtained via the GSEA 4.0.1 software (39). Based on the ranking of normalized enrichment scores, the top 10 terms were displayed to identify differences in biological pathways and behaviors between high- and low-risk patients.

\section{DNA Methylation Analysis}

Illumina Human Methylation $450 \mathrm{k}$ data were download from the UCSC Xena website. Using the "ChAMP" package, we filtered the non-cg probes; the CpG falls near a singlenucleotide polymorphism; the probe aligns to multiple locations; and from the X and Y chromosomes (40). Subsequently, the remaining probes were used to conduct the differential analysis between high- and low-risk patients. The mean methylation value of the differential probes was utilized to display the methylation of the promoter, body, $3^{\prime}$ untranslated regions ( $3^{\prime} \mathrm{UTR}$ ), and intergenic regions (IGR) in high- and low-risk patients. Furthermore, the association between methylation and prognosis was investigated. Finally, the differential probes were enriched using the "missMethyl" package to observe potential mechanisms involved in different prognoses. 


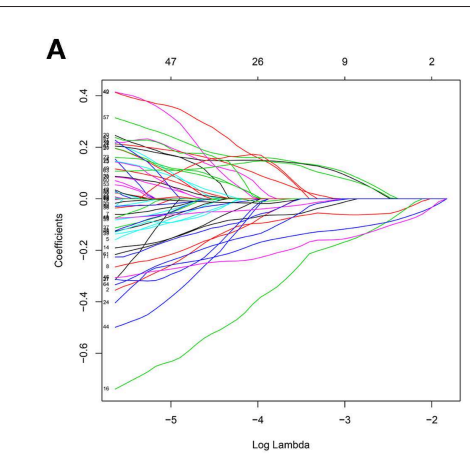

D

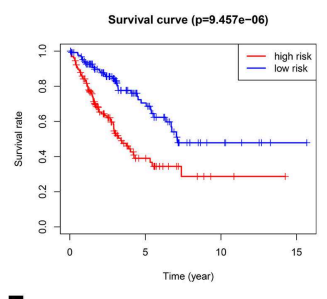

E

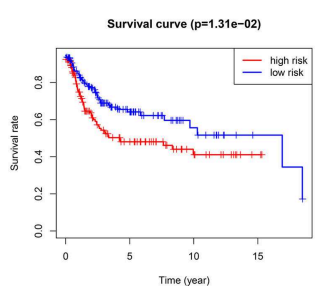

B

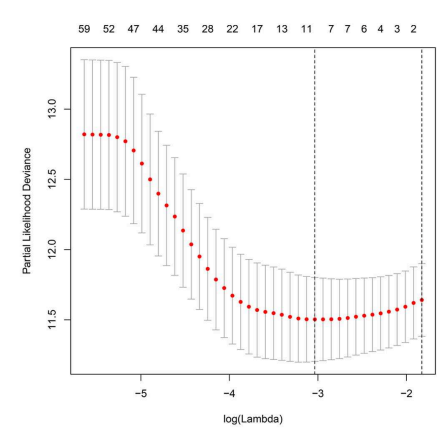

$\mathbf{F}$
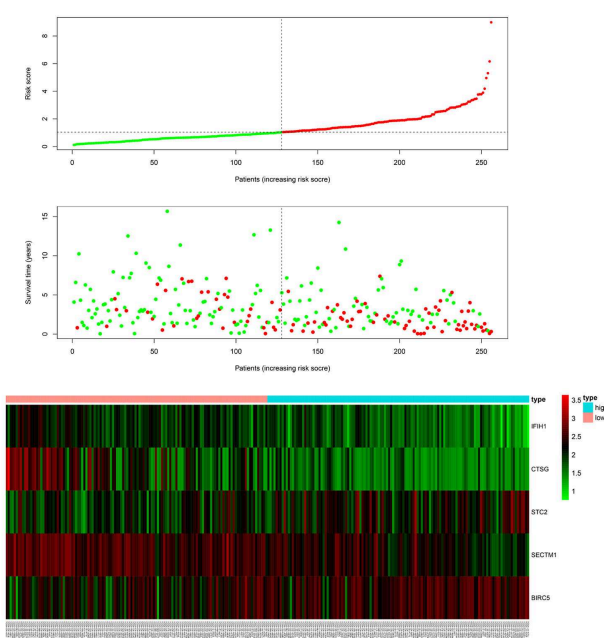

C

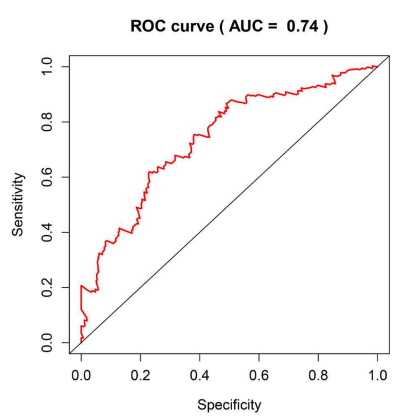

G
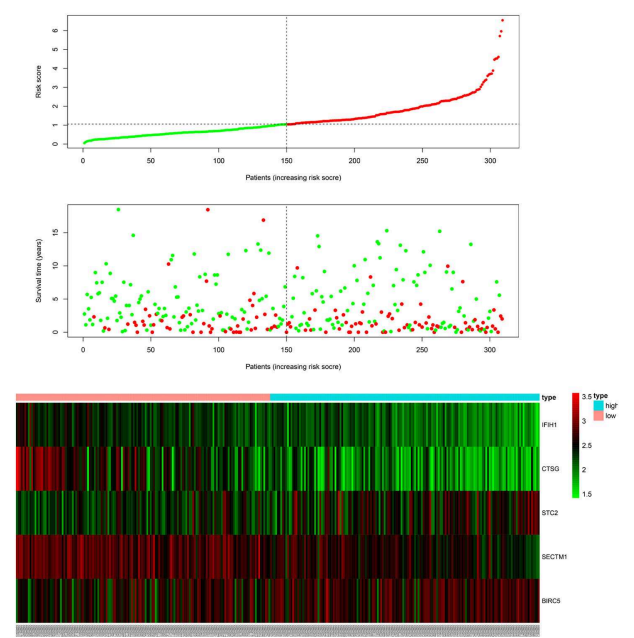

FIGURE 3 | Construction, validation, and assessment of the prognostic model. (A,B) Identification of prognostic genes using the least absolute shrinkage and selection operator (LASSO) regression analysis. (A) Coefficients of the LASSO regression analysis. (B) Selection of tuning parameters based on the 1000 cross-validations method. (C) The receiver operating characteristic (ROC) curves of the prognostic model in TCGA dataset. The survival curve for high- and low-risk patients in TCGA (D) and GSE21050 (E) datasets. Differences in risk score, survival time, and gene expression of the optimal immune-related prognostic genes (IRPGs) between high- and low-risk patients from TCGA (F) and GSE21050 (G) datasets.

\section{Identification of Independent Prognostic Factors (IPF) for STS}

Clinical factors (i.e., age, sex, margin status, and metastatic diagnosis), mitotic counts, total necrosis percent of tumors, risk scores, and immune scores were included in the univariate and multivariate Cox regression analyses to further evaluate the prognostic model and identify IPF. Only factors with $p<0.05$ in both analyses were considered IPF. In addition, the prognostic ability of risk scores and immune scores was evaluated via ROC curves.

\section{RESULTS}

\section{Collection and Preprocessing of Gene Expression and Clinical Data}

The datasets of TCGA contained 263 samples with STS and two matched controls. After removing the two matched controls, the gene expression data were analyzed. The GSE21050 dataset
TABLE 1 | The multivariate Cox regression analysis of genes for overall survival.

\begin{tabular}{cccccc}
\hline Genes & \multicolumn{5}{c}{ Overall survival } \\
\cline { 2 - 5 } & coef & HR & \multicolumn{2}{c}{$\mathbf{9 5 \%} \mathbf{~ C l}$} & $\boldsymbol{p}$-value \\
\hline IFIH1 & -0.373 & 0.689 & 0.505 & 0.939 & 0.018 \\
CTSG & -0.215 & 0.806 & 0.671 & 0.968 & 0.021 \\
STC2 & 0.195 & 1.215 & 1.027 & 1.438 & 0.023 \\
SECTM1 & -0.259 & 0.772 & 0.631 & 0.944 & 0.012 \\
BIRC5 & 0.229 & 1.258 & 1.033 & 1.530 & 0.022 \\
\hline
\end{tabular}

coef, coefficient; HR, hazard ratio; Cl, confidence interval.

comprised 310 samples with STS. Using a preliminary filter, 4,113 and 5,046 genes with top 25\% variances were obtained from TCGA and GEO datasets, respectively. Notably, they shared 2,447 genes with the WGCNA. The corresponding clinical data of patients were matched to their corresponding gene expression profiles for the subsequent analysis. A total of 256 patients with 


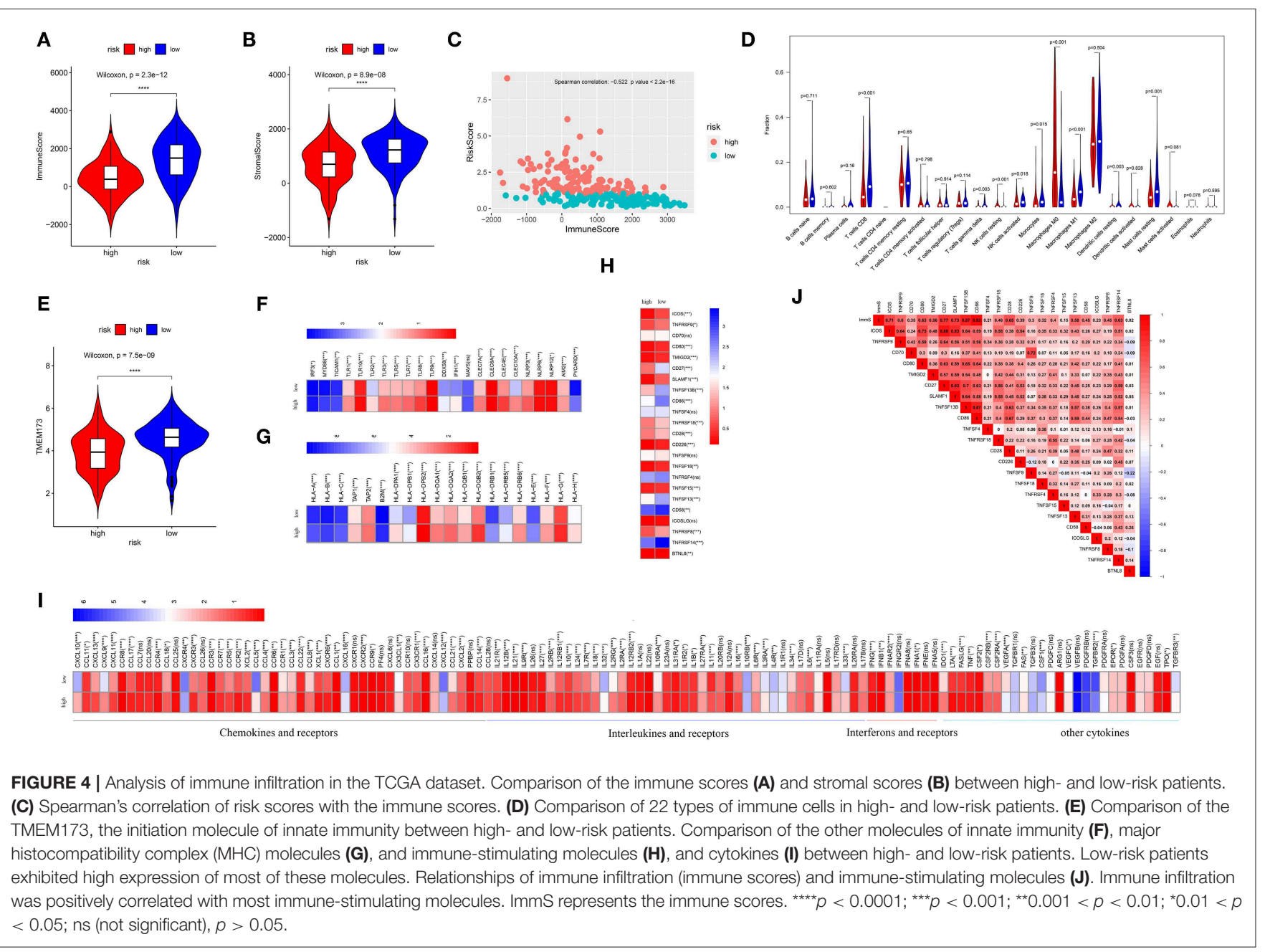

survival information were included in the survival or prognosis analysis in TCGA dataset. The patient (GSM525864) lacking information regarding survival and metastasis were not included in these analyses of the GSE21050 dataset.

\section{Two Modules Containing 1,141 Genes Were Associated With Survival}

As shown in Figure 1A, an adjacency matrix was constructed based on the soft threshold power $\beta$ (optimal $\beta=3 ; R^{2}=0.9$ ), determined through the scale-free topology criterion. Figure 1B illustrates the identified modules obtained using the Dynamic Tree Cut method. Subsequently, the identified modules were related to the clinical data. Compared with other modules, two modules were highly associated with survival (Figure 1C). The blue module significantly influenced the survival time of patients with STS (Pearson's correlation between blue module and the trait of survival time: $0.11, p=0.08$ ). A similar relationship was observed in the brown module associated with the OS status (Pearson's correlation between brown module and the trait of OS status: $0.14, p=0.03$ ). Furthermore, genes in the blue and brown modules exhibited high positive correlations with survival
(Pearson's correlation between gene significance and module membership: $0.41, p=5.7 \mathrm{e}-39$; Pearson's correlation between gene significance and module membership: $0.46, p=1.7 \mathrm{e}-12$, respectively) (Figures 1D,E). Function annotation revealed that 929 and 212 genes in the blue module and brown module were mainly involved in the immune process and cell cycle process, respectively. These findings indicated that genes in these two modules highly influenced survival in STS $(p<0.05)$ (Figure S1).

\section{Identification and Validation of IGRPM}

The 1,141 genes obtained from the WGCNA and the IRGs from the ImmPort database shared 207 IRGs, which were involved in immune activity via multiple pathways $(p<0.05)$ (Figure 2 ). These 207 IRGs were subsequently analyzed to identify optimal IRPGs. Firstly, 81 IRGs associated with survival from univariate Cox regression analysis were obtained based on $p<0.05$. LASSO regression analysis displayed that nine IRGs (i.e., CD1C, C-X$\mathrm{C}$ motif chemokine ligand 2 [CXCL2], interferon induced with helicase C domain 1 [IFIH1], cathepsin G [CTSG], stanniocalcin 2 [STC2], secreted and transmembrane 1 [SECTM1], baculoviral IAP repeat containing 5 [BIRC5], endothelin 3 [EDN3], and nuclear receptor subfamily 1 group $\mathrm{H}$ member 3 [NR1H3]) 

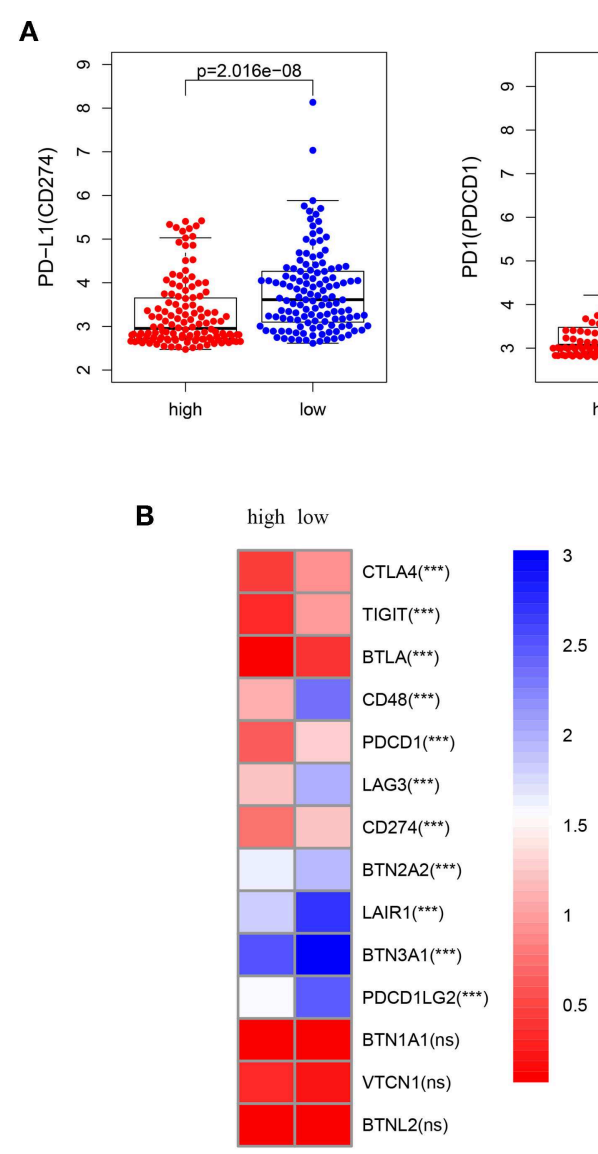
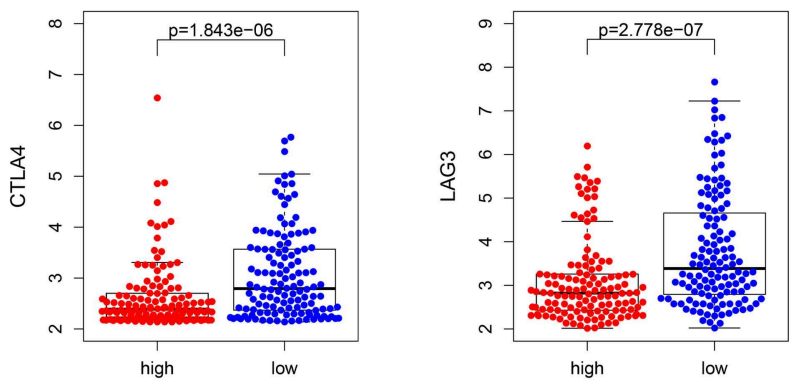

c

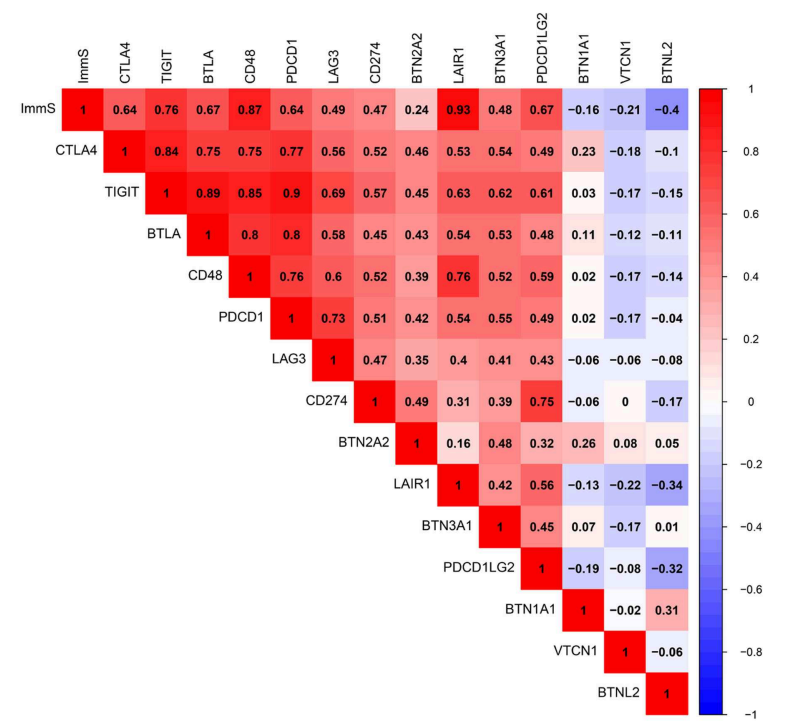

FIGURE 5 | Inhibition of immunity in low-risk patients from TCGA dataset. (A) Differences in common immune checkpoint molecules between high- and low-risk patients. (B) Differences in immune-inhibiting molecules between high- and low-risk patients. (C) Relationships of immune infiltration (immune scores) and immune-inhibiting molecules. Immune infiltration was positively correlated with most immune-inhibiting molecules. ImmS represents the immune scores. ${ }^{\star * \star} p<0.001$; ns (not significant), $p>0.05$.

were associated with survival based on the 1000 cross-validations approach (Figures 3A,B). Five optimal IRPGs (i.e., IFIH1, CTSG, STC2, SECTM1, and BIRC5) were obtained from the multivariate Cox regression analysis (Table 1). Subsequently, we used the "predict" function to construct the prognostic model with an area under the curve of 0.74 in 5-year survival rates (Figure 3C). High- and low-risk patients, determined according to their risk scores, had significant differences in survival rates in TCGA $(p=9.457 \mathrm{e}-06)$ (Figure 3D) and GSE21050 $(p=1.31 \mathrm{e}-02)$ datasets (Figure 3E). The prognostic model was further assessed and validated (Figures 3F,G).

\section{Relatively Higher Immune Activation Was Observed in Low-Risk Patients}

Estimated immune and stromal scores, immune cells, and immune-related molecules were assessed for the level of immune infiltration between high- and low-risk patients. Higher immune scores and stromal scores were observed in low-risk patients in TCGA $(p=2.3 \mathrm{e}-12 ; p=8.9 \mathrm{e}-08$, respectively) (Figures 4A,B) and GSE21050 ( $p=2.9 \mathrm{e}-16 ; p=6.4 \mathrm{e}-15$, respectively) (Figures S2A,B) datasets. Strong negative correlations between the immune scores and risk scores indicated that higher immune filtration was a potential protective factor for survival (Spearman's correlation: $-0.522, p<2.2 \mathrm{e}-16$; Figure $4 \mathrm{C}$ and Spearman's correlation: $-0.540, p<2.2 \mathrm{e}-16$; Figure S2C). By analyzing the proportion of immune cells in TCGA dataset, a greater number of immune cells (i.e., CD8 T cells, gamma delta T cells, activated natural killer (NK) cells, monocytes, M1 macrophages, and resting mast cells) were found in low-risk patients $(p<0.05$, Figure 4D). Similarly, more CD8 T cells, follicular helper T cells, gamma delta T cells, activated NK cells, M1 macrophages, and resting mast cells were observed in lowrisk patients in the GES21050 dataset ( $p<0.05$, Figure S2D). In addition, immune-related molecules demonstrated that lowrisk patients had higher immune activation. High expression of innate immune modules, especially TMEM173(STING1) (Wilcoxon rank-sum test: $p=7.5 \mathrm{e}-09$, Figure $4 \mathrm{E}$ and $p=4.8 \mathrm{e}-09$, Figure S2E), was noted in low-risk patients 


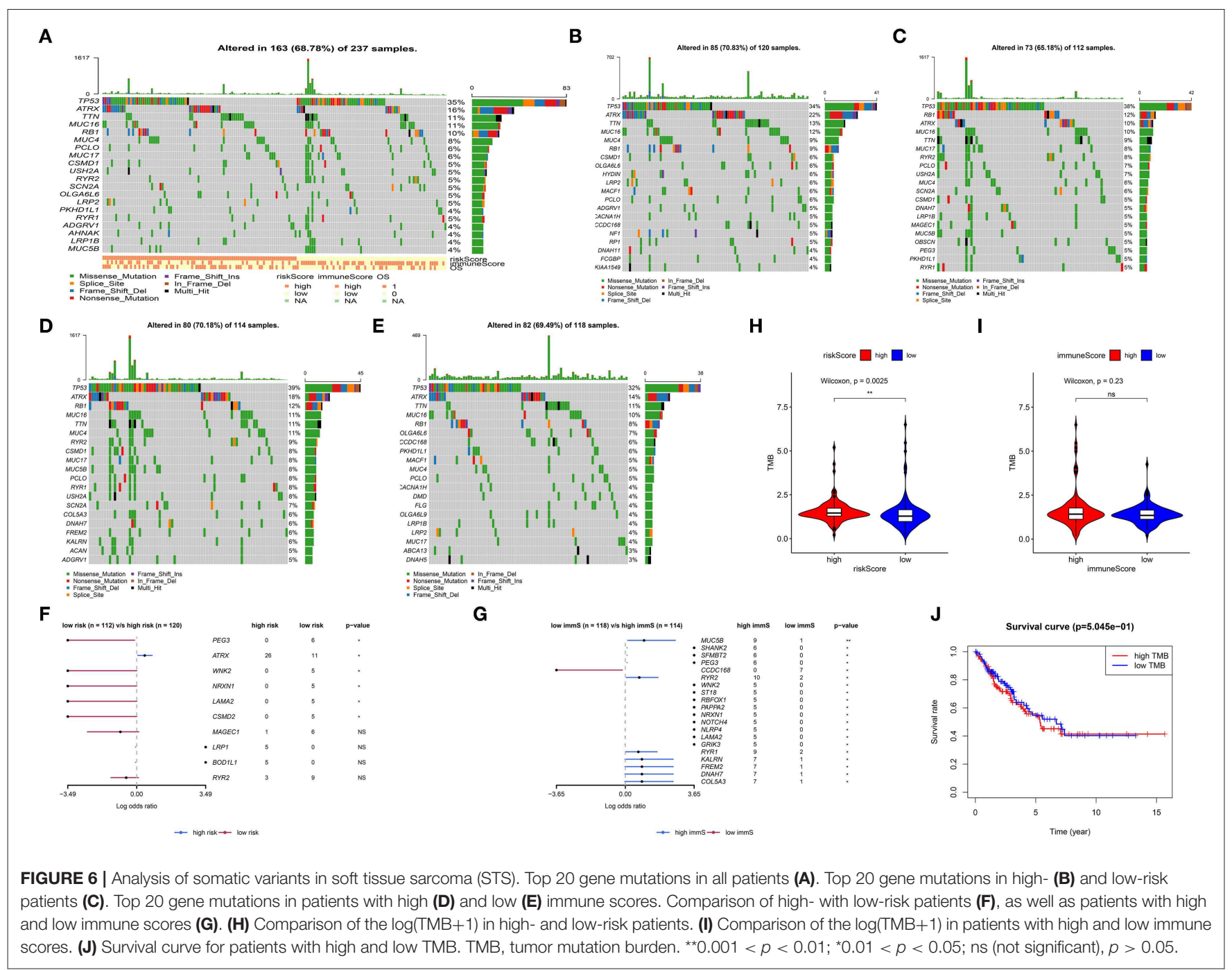

(Figure 4F, Figure S2F). Moreover, low-risk patients exhibited stronger antigen-presenting capacity, mainly measured through the higher expression of major histocompatibility complex (MHC) molecules (e.g., MHC I, MHC II, etc.) (Figure 4G, Figure S2G). A similar tendency was observed in comparisons of immune-stimulating molecules, cytokines, and corresponding receptors between patients with different risks (Figures $4 \mathbf{H}, \mathbf{I}$, Figures S2H,I). Finally, positive correlations between immune scores and immune-stimulating molecules further supported and explained the above findings (Figure 4J, Figure S2J).

\section{Low-Risk Patients Showed Immunosuppression}

Characterized by higher immune activation, low-risk patients from TCGA also showed immunosupression. Immunosuppression in low-risk patients was verified through immune-inhibiting molecules, especially common immune checkpoints, such as PD-L1 (CD274), PD1 (programmed cell death 1 [PDCD1]), cytotoxic T-lymphocyte associated protein 4 [CTLA4], and lymphocyte activating 3 [LAG3] (Figures 5A,B,
Figures S3A,B). There were no differences in the expression level of these four immune checkpoints between the sexes (Figure S4). In addition, higher levels of inhibiting cytokines (e.g., interleukin 10) were present in the tumor microenvironment of low-risk patients (Figure 4I, Figure S2I). The strong correlations noted between immune scores and most immune-inhibiting molecules revealed that low-risk patients exhibited higher immune infiltration and immunosuppression, which increased the risk of immune escape of STS (Figure 5C, Figure S3C).

\section{Analysis of Somatic Variants}

Gene mutations in STS were also studied. As shown in Figure 6A, $68.78 \%$ of the top 20 mutations occurred in all patients, with missense mutation being the most common type. Of note, different types of mutations were observed between high- and low-risk patients. Tumor protein p53 (TP53), ATRX, titin (TTN), mucin 16 (MUC16), and RB transcriptional corepressor 1 (RB1) were the most commonly mutated genes ( $>10 \%$ mutation rate). Obvious differences in the top 20 mutated genes between highand low-risk patients are illustrated in Figures 6B,C. Following 

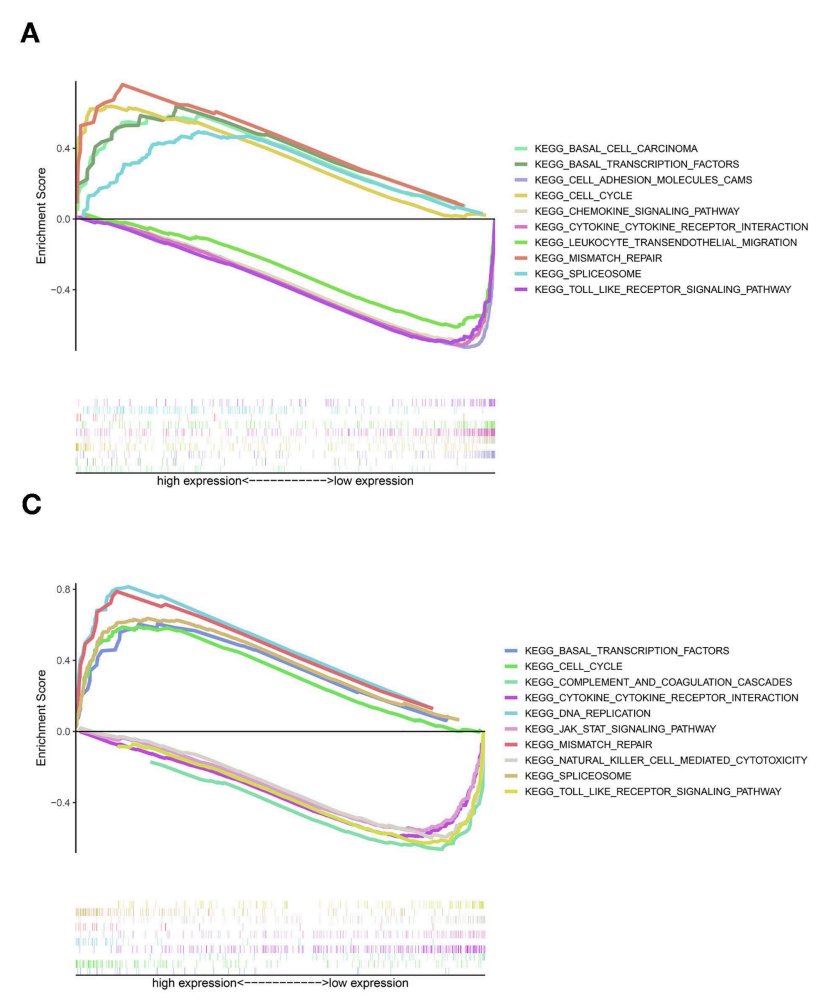

B
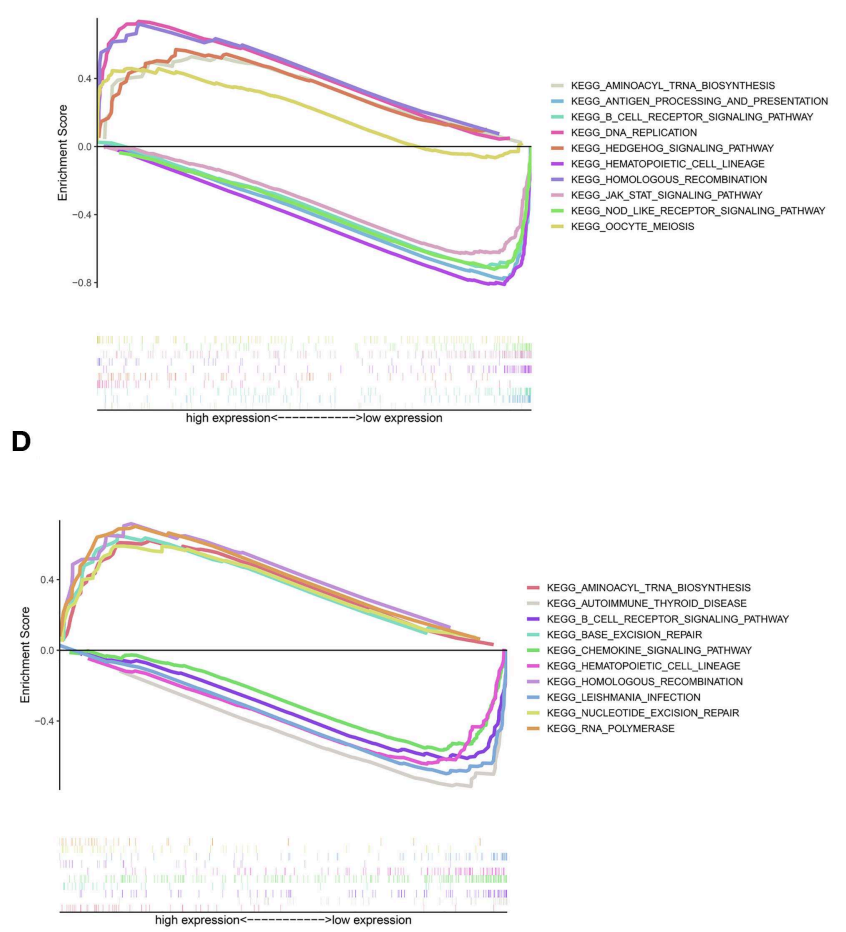

FIGURE 7 | Gene set enrichment analysis for high- and low-risk patients. (A) Top 1-5 terms of the GSEA from TCGA dataset. (B) Top 6-10 terms of the GSEA from TCGA dataset. (C) Top 1-5 terms of the GSEA from the GSE21050 dataset. (D) Top 6-10 terms of the GSEA from the GSE21050 dataset.

the assignment of patients based on the median of immune scores, more top 20 mutated genes were observed in those with high immune scores (Figures 6D,E). Figures 6F,G show the comparison of high- and low-risk patients, as well as those with high- and low-immune scores. ATRX, paternally expressed 3 (PEG3), WNK lysine deficient protein kinase 2 (WNK2), neurexin 1 (NRXN1), laminin subunit alpha 2 (LAMA2), and CUB and Sushi multiple domains 2 (CSMD2) demonstrated significant gene mutation differences between high- and low-risk patients $(p<0.05)$ (Figure 6F). Highly mutated genes, such as PEG3, WNK2, NRXN1, and LAMA2 in low-risk patients were also present in patients with high immune scores (Figure 6G). Figure 6H displays that high-risk patients had more TMB $(p=0.0025)$. However, TMB was not associated with OS in STS (Figure 6J). In addition, more TMB was observed in patients with high immune scores; however, the difference was not significant $(p=0.23)$ (Figure 6I).

\section{Potential Mechanisms Associated With Prognosis}

GSEA revealed that different pathways were altered in highand low-risk patients, and demonstrated potential mechanisms associated with the biological phenotype (Figure 7). According to the ranking of normalized enrichment scores, KEGG pathways (e.g., cell cycle, mismatch repair, basal transcription factors, spliceosome, aminoacyl tran biosynthesis, and DNA replication) were activated in high-risk patients with shorter OS (Figure 7). However, the activation of immune-related pathways (e.g., the toll-like receptor signaling pathway, Janus kinase/STAT signaling pathway, cytokine-cytokine receptor interaction, B cell receptor signaling pathway, and natural killer cell-mediated cytotoxicity) led to better prognosis in low-risk patients (Figure 7).

\section{High Relative Methylation Was Observed in Low-Risk Patients}

After removing the low-quality probes, differential methylation probes were obtained based on the adjusted $p<0.05$. Subsequently, we extracted the differential probes in the promoter, body, 3'UTR, and IGR. Figure 8A shows that high relative methylation of these four genomic regions was detected in low-risk patients. Notably, high methylation was associated with better OS (Figure 8B). The differential probes were mainly involved in neuroactive ligand-receptor interaction, RAP1 signaling pathway, ECM-receptor interaction, immune-related pathway (e.g., T cell receptor signaling pathway and cytokinecytokine receptor interaction) and pathways in cancer (e.g., Ras signaling pathway, breast cancer, and basal cell carcinoma) (Table 2). 


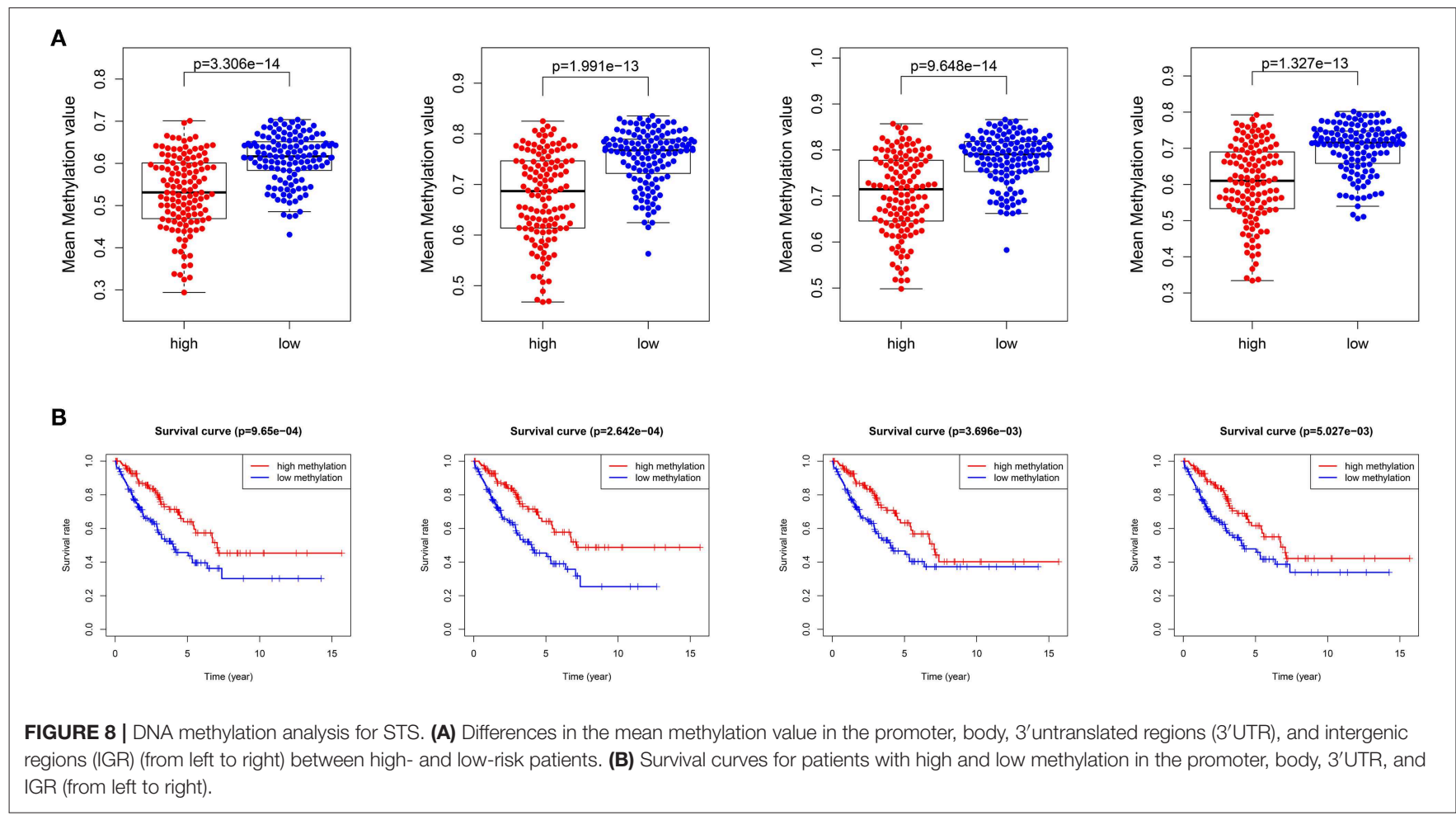

\section{Age, Mitotic Count, and Risk Scores May Be IPF in STS}

Figure S5A shows that age $(p=0.004)$, margin status $(p=$ $0.009)$, diagnosis of metastasis $(p<0.001)$, mitotic count $(p<$ $0.01)$, immune scores $(p=0.021)$, and risk scores $(p<0.001)$ were prognostic factors identified in the univariate analysis. The level of immune infiltration measured using the immune scores affected survival in STS. The age, mitotic count and risk scores were considered IPF for STS (Figures S5A,B). Figure S6 shows that risk scores (area under the curve:0.74) had better prognostic ability than immune scores (area under the curve:0.388).

\section{DISCUSSION}

The application of immunotherapy to multiple cancers significantly improved the survival of patients (41). Based on the accumulating evidence and concept of immunotherapy, this approach is effective in the treatment of advanced or metastatic cancers. Immunotherapy has been utilized for the treatment of STS. Previous studies demonstrated that partial patients with STS could benefit from inhibition of PD-1 $(42,43)$. Immune-related signatures such as tumor inflammation signature (44), immunological constant of rejection (45), and immunophenoscore (46) have been demonstrated as predictors for prognosis and response to immunotherapy in tumors. However, A pan-cancer study revealed that the score of tumor inflammation signature was not associated with prognosis of sarcoma based on univariate Cox regression analysis (44). Therefore, the discovery of new predictive biomarkers of patient response and comprehensive studies of tumor immune microenvironment in STS are crucial for the optimization of immunotherapy in STS (42).

Multi-omic data were utilized to identify potential IRPGs, as well as construct and validate the IGRPM based on WGCNA, univariate, LASSO, and multivariate regression analyses. Finally, five optimal IRPGs and one IGRPM were determined and validated through two datasets involving 573 patients. The IGRPM based on the five IRPGs demonstrated satisfactory performance in predicting the survival rates and risk stratification in patients with STS. IFIH1 (also termed melanoma differentiation-associated gene 5) exhibits an antitumor effect (47) and was a protective gene in STS. CTSG, which possesses the ability to enhance the cytotoxicity of human natural killer cells (48) was overexpressed in low-risk patients with better OS compared with high-risk patients. STC2 and BIRC5 promote metastasis and progression in different types of cancer [e.g., head and neck squamous cell carcinoma (49), hepatocellular carcinoma (50), lung cancer (51), and ovarian tumor (52)] and were overexpressed in high-risk patients. SECTM1, the stimulator of $\mathrm{T}$ cells (53), was also identified as a protective gene. Overall, the different prognoses between high- and lowrisk patients was consistent with the expression of the five IRPGs, validating the accuracy of the IRGs and IGRPM obtained in this study. To our knowledge, these five IRPGs were firstly combined to construct the IGRPM for STS.

Characterization of tumor immune microenvironment was performed based on two algorithms of ESTIMATE and CIBERSORT. We demonstrated that longer OS in low-risk patients was associated with higher immune activation (including 
TABLE 2 | The KEGG pathways for the differential probes of methylation.

\begin{tabular}{|c|c|}
\hline Pathway & FDR \\
\hline Neuroactive ligand-receptor interaction & 3.40E-05 \\
\hline Rap1 signaling pathway & $7.58 \mathrm{E}-05$ \\
\hline ECM-receptor interaction & 0.000226155 \\
\hline Calcium signaling pathway & 0.000324435 \\
\hline Olfactory transduction & 0.000668637 \\
\hline Bile secretion & 0.001056711 \\
\hline Pathways in cancer & 0.001266947 \\
\hline Cytokine-cytokine receptor interaction & 0.001669184 \\
\hline Adrenergic signaling in cardiomyocytes & 0.001669184 \\
\hline Phospholipase D signaling pathway & 0.002095849 \\
\hline cAMP signaling pathway & 0.003042019 \\
\hline Glutamatergic synapse & 0.003538057 \\
\hline PI3K-Akt signaling pathway & 0.005381894 \\
\hline Inflammatory mediator regulation of TRP channels & 0.006788386 \\
\hline Melanogenesis & 0.006788386 \\
\hline Ras signaling pathway & 0.008512401 \\
\hline Axon guidance & 0.008790691 \\
\hline C-type lectin receptor signaling pathway & 0.008790691 \\
\hline Dopaminergic synapse & 0.008790691 \\
\hline Estrogen signaling pathway & 0.008790691 \\
\hline Vascular smooth muscle contraction & 0.009496407 \\
\hline Hypertrophic cardiomyopathy (HCM) & 0.009632625 \\
\hline Proteoglycans in cancer & 0.00988492 \\
\hline Basal cell carcinoma & 0.010425995 \\
\hline Arachidonic acid metabolism & 0.011721194 \\
\hline Oxytocin signaling pathway & 0.011721194 \\
\hline Dilated cardiomyopathy (DCM) & 0.011721194 \\
\hline MAPK signaling pathway & 0.01188034 \\
\hline Circadian entrainment & 0.01188034 \\
\hline Gastric cancer & 0.012601185 \\
\hline Focal adhesion & 0.016998021 \\
\hline Renin secretion & 0.017058953 \\
\hline Linoleic acid metabolism & 0.018485501 \\
\hline Insulin resistance & 0.018728758 \\
\hline Human papillomavirus infection & 0.018728758 \\
\hline Tight junction & 0.019045102 \\
\hline Glycerophospholipid metabolism & 0.019618676 \\
\hline Wnt signaling pathway & 0.019855177 \\
\hline Hippo signaling pathway & 0.019855177 \\
\hline cGMP-PKG signaling pathway & 0.022978288 \\
\hline Platelet activation & 0.022978288 \\
\hline AGE-RAGE signaling pathway in diabetic complications & 0.022978288 \\
\hline Breast cancer & 0.025542783 \\
\hline Ether lipid metabolism & 0.025994303 \\
\hline Relaxin signaling pathway & 0.025994303 \\
\hline Regulation of actin cytoskeleton & 0.027185869 \\
\hline Amyotrophic lateral sclerosis (ALS) & 0.027202385 \\
\hline Gastric acid secretion & 0.028582194 \\
\hline Insulin secretion & 0.029401081 \\
\hline Apelin signaling pathway & 0.034688902 \\
\hline
\end{tabular}

(Continued)
TABLE 2 | Continued

\begin{tabular}{ll}
\hline Pathway & FDR \\
\hline Phototransduction & 0.038659036 \\
Sphingolipid signaling pathway & 0.039963306 \\
Pertussis & 0.040982726 \\
Parathyroid hormone synthesis, secretion and action & 0.042635342 \\
Aldosterone synthesis and secretion & 0.045523293 \\
T cell receptor signaling pathway & 0.04895089 \\
Fc epsilon RI signaling pathway & 0.04895089 \\
Adipocytokine signaling pathway & 0.04895089 \\
\hline
\end{tabular}

KEGG, kyoto encyclopedia of genes and genomes; FDR, false discovery rate.

innate and adaptive immunity). Higher immune scores and more activated immune cell infiltrations (e.g., CD8 T cells and activated NK cells) $(54,55)$ supported this notion and a similar phenomenon was also present in other types of cancer $(15,56)$. Specifically, low-risk patients had a greater number of innate immune cells owing to the higher expression of TMEM173, triggering innate immunity and innate immunityrelated molecules (57). These findings were consistent with those obtained from the algorithm of CIBERSORT. The higher immune activation was also induced by the high expression of immune-stimulating modules [e.g., inducible $\mathrm{T}$ cell costimulator [ICOS] (58) and CD80 (59)] and cytokines (e.g., C-C motif chemokine ligand 4 [CCL4], CXCL9, and CXCL10) (60). In addition, the MHC molecules promoted antigen presentation in low-risk patients. Therefore, the lack of immune cells, immunestimulating molecules, cytokines, and weak antigen-presenting ability led to poor prognosis in high-risk patients.

Immune escape also affects survival (61) and response to immunotherapy in patients with cancer. In this study, tumor cells tended to escape the immune system due to inhibition of immunity in low-risk patients. This effect was demonstrated by high expression of immune inhibiting molecules (e.g., common immune checkpoints and inhibiting cytokines). The expression of immune checkpoints in tumor cells negatively regulates $\mathrm{T}$ cells and evades immune killing (62). The high expression of immune checkpoint molecules further suggested that the five identified IRPGs are predictive biomarkers of response to immunotherapy. A recent study reported sex-dependent differences in patient response to immunotherapy in melanoma and non-small-cell lung cancer $(41,63)$. However, comparison of common checkpoints PD-L1 (CD274), PD1 (PDCD1), CTLA4, and LAG3 between male and female patients reveled that sex is not the main factor for the prediction of response to immune checkpoint inhibitors in STS. This finding was similar to the results reported in a recent study (64). Inhibition of cytokines (e.g., IL10) (45) also inhibited immunity in low-risk patients.

TMB is an emerging biomarker for predicting the effect of immunotherapy in multiple types of cancer (65). The different top 20 gene mutations observed in patients grouped according to risk scores and immune scores led to the hypothesis that low-risk patients may carry more gene mutations. However, this hypothesis was not validated by the value of the TMB. 
We revealed that high-risk patients (low immune infiltration) had high TMB. This was not consistent with the theory that high mutations (TMB) tend to generate neoantigen epitopes. We hypothesized that high TMB and low antigen-presenting capacity caused low immune infiltration in high risk patients. Of note, both high- and low-risk patients had relative low TMB, which was consistent with a previous study (66). This indicated that the TMB was not suitable for the prediction of response to immunotherapy in STS. Furthermore, the TMB was not associated with survival in STS.

The GSEA revealed that pathways related to cell cycle, DNA replication, and cancer were activated in high-risk patients, leading to poor prognosis. However, the activation of immune-related pathways improved survival. These results also validated the IRPGs and the IGRPM. Furthermore, differences in DNA methylation were investigated between high- and lowrisk patients. Relative low methylation in high-risk patients contributed to poor prognosis. The KEGG analysis further revealed the underlying mechanism involved in the effect of DNA methylation on prognosis. In addition, age, mitotic count and risk score were IPF in STS, further validating the prognostic model.

This study had the following clinical implications and strengths. Firstly, although the use of immunotherapy benefited the treatment of cancers, the low response linked to this therapeutic approach limited its use. The identification of "hot tumors" and transformation of "cold tumors" to "hot tumors" could overcome the current predicament (60). Five IRPGs with satisfactory performance in the discrimination of high risk ("cold tumors") and low risk ("hot tumors") were identified as potential predictive biomarkers of response to immunotherapy. Low-risk patients not only showed high immune activation but also had inhibition of immunity, especially high expression of checkpoint molecules. Secondly, an increasing number of studies reported that expression of the MHC could predict response to immune checkpoint blockade (67). The significant differences in MHC expression between high- and low-risk patients also suggested the potential use of the five aforementioned IRPGs for the prediction of response to immunotherapy. Thirdly, the differences between high- and low-risk patients revealed by the multi-omic analysis may provide a reference for subsequent studies on the transformation of "cold tumors" to "hot tumors" or improvement of treatment of STS. Fourthly, the combination of different bioinformatics methods increased the reliability of the results.

However, this study was also characterized by some limitations. Firstly, the small sample sizes of the different histological subtypes limited the integrated analysis for each

\section{REFERENCES}

1. Stiller CA, Trama A, Serraino D, Rossi S, Navarro C, Chirlaque MD, et al. Descriptive epidemiology of sarcomas in Europe: report from the RARECARE project. Eur J Cancer. (2013) 49:684-95. doi: 10.1016/j.ejca.2012. 09.011 type. Secondly, the stage of STS, which was not available in TCGA dataset, also had a significant impact on prognosis. Subsequent studies focusing on different histological subtypes and the stage of STS are warranted to validate the results of the present study. Thirdly, the ability of five IRPGs to predict prognosis and response to immunotherapy could not be assessed by current methods such as using PD-L1 immunohistochemistry, or Nanostring tumor inflammation signature for lack of data in STS (68). Therefore, five IRPGs also need to be tested in basic experiment and clinical trials.

\section{CONCLUSION}

In this study, one IGRPM with independent prognostic ability based on five optimal IRPGs (i.e., IFIH1, CTSG, STC2, SECTM1, and BIRC5) were identified and validated in STS. Through the comprehensive study of the tumor immune microenvironment, we demonstrated that these five IRPGs contributed to risk stratification and the identification of patients who are responsive to immunotherapy. Furthermore, the multi-omic analysis revealed the potential mechanisms affecting prognosis, providing additional references for the treatment of STS. More studies focusing on histological subtype were needed to provide more precise treatment.

\section{DATA AVAILABILITY STATEMENT}

The datasets presented in this study can be found in online repositories. The names of the repository/repositories and accession number(s) can be found in the article/ Supplementary Material.

\section{AUTHOR CONTRIBUTIONS}

H-YG, R-XW, and CZ conceived and designed the study. $\mathrm{R}-\mathrm{XW}, \mathrm{H}-\mathrm{YG}$, and L-LL performed the analysis procedures. $\mathrm{H}-\mathrm{YG}, \mathrm{MY}, \mathrm{H}-\mathrm{CZ}$, and CZ analyzed the results. H-YG, MY, and $\mathrm{CZ}$ contributed analysis tools. H-YG, L-LL, CZ, and R-XW contributed to the writing of the manuscript. H-YG, R-XW, and $\mathrm{CZ}$ performed project administration. All authors reviewed the manuscript.

\section{SUPPLEMENTARY MATERIAL}

The Supplementary Material for this article can be found online at: https://www.frontiersin.org/articles/10.3389/fonc. 2020.01317/full\#supplementary-material 
4. Smith HG, Memos N, Thomas JM, Smith MJ, Strauss DC, Hayes AJ. Patterns of disease relapse in primary extremity soft-tissue sarcoma. Br J Surg. (2016) 103:1487-96. doi: 10.1002/bjs.10227

5. Casali PG, Jost L, Sleijfer S, Verweij J, Blay JY. Soft tissue sarcomas: ESMO clinical recommendations for diagnosis, treatment and follow-up. Ann Oncol. (2009) 20(Suppl. 4):132-6. doi: 10.1093/annonc/mdp153

6. Casali PG, Abecassis N, Aro HT, Bauer S, Biagini R, Bielack S, et al. Soft tissue and visceral sarcomas: ESMO-EURACAN clinical practice guidelines for diagnosis, treatment and follow-up. Ann Oncol. (2018) 29(Suppl. 4):iv51-67. doi: 10.1093/annonc/mdy096

7. Italiano A, Le Cesne A, Mendiboure J, Blay JY, Piperno-Neumann S, Chevreau C, et al. Prognostic factors and impact of adjuvant treatments on local and metastatic relapse of soft-tissue sarcoma patients in the competing risks setting. Cancer. (2014) 120:3361-9. doi: 10.1002/cncr. 28885

8. Sevic I, Spinelli FM, Cantero MJ, Reszegi A, Kovalszky I, Garcia MG, et al. The role of the tumor microenvironment in the development and progression of hepatocellular carcinoma. In: JEE Tirnitz-Parker, editor. Hepatocellular Carcinoma. Brisbane, QLD: Codon Publications. (2019). p. 29-45.

9. Jiang Y, Zhang Q, Hu Y, Li T, Yu J, Zhao L, et al. Immuno score signature: a prognostic and predictive tool in gastric cancer. Ann Surg. (2018) 267:504-13. doi: 10.1097/SLA.0000000000002116

10. Jiang X, Wang J, Deng X, Xiong F, Ge J, Xiang B, et al. Role of the tumor microenvironment in PD-L1/PD-1-mediated tumor immune escape. $\mathrm{Mol}$ Cancer. (2019) 18:10. doi: 10.1186/s12943-018-0928-4

11. Schachter J, Ribas A, Long GV, Arance A, Grob JJ, Mortier L, et al. Pembrolizumab versus ipilimumab for advanced melanoma: final overall survival results of a multicentre, randomised, openlabel phase 3 study (KEYNOTE-006). Lancet. (2017) 390:1853-62. doi: 10.1016/S0140-6736(17)31601-X

12. Topalian SL, Sznol M, McDermott DF, Kluger HM, Carvajal RD, Sharfman $\mathrm{WH}$, et al. Survival, durable tumor remission, and long-term safety in patients with advanced melanoma receiving nivolumab. J Clin Oncol. (2014) 32:102030. doi: 10.1200/JCO.2013.53.0105

13. Saller J, Walko CM, Millis SZ, Henderson-Jackson E, Makanji R, Brohl AS. Response to checkpoint inhibitor therapy in advanced classic kaposi sarcoma: a case report and immunogenomic study. J Natl Compr Canc Netw. (2018) 16:797-800. doi: 10.6004/jnccn.2018.7018

14. Papadopoulos KP, Romero RS, Gonzalez G, Dix JE, Lowy I, Fury M. Anti-Hu-associated autoimmune limbic encephalitis in a patient with PD-1 inhibitor-responsive myxoid chondrosarcoma. Oncologist. (2018) 23:118-20. doi: 10.1634/theoncologist.2017-0344

15. Yang S, Wu Y, Deng Y, Zhou L, Yang P, Zheng Y, et al. Identification of a prognostic immune signature for cervical cancer to predict survival and response to immune checkpoint inhibitors. Oncoimmunology. (2019) 8:e1659094. doi: 10.1080/2162402X.2019.1659094

16. Song Q, Shang J, Yang Z, Zhang L, Zhang C, Chen J, et al. Identification of an immune signature predicting prognosis risk of patients in lung adenocarcinoma. J Transl Med. (2019) 17:70. doi: 10.1186/s12967-019-1824-4

17. Zeng D, Li M, Zhou R, Zhang J, Sun H, Shi M, et al. Tumor microenvironment characterization in gastric cancer identifies prognostic and immunotherapeutically relevant gene signatures. Cancer Immunol Res. (2019) 7:737-50. doi: 10.1158/2326-6066.CIR$18-0436$

18. Chibon F, Lagarde P, Salas S, Perot G, Brouste V, Tirode F, et al. Validated prediction of clinical outcome in sarcomas and multiple types of cancer on the basis of a gene expression signature related to genome complexity. Nat Med. (2010) 16:781-7. doi: 10.1038/nm.2174

19. Gautier L, Cope L, Bolstad BM, Irizarry RA. affy-analysis of Affymetrix GeneChip data at the probe level. Bioinformatics. (2004) 20:307-15. doi: 10.1093/bioinformatics/btg405

20. Gu Y, Lu L, Wu L, Chen H, Zhu W, He Y. Identification of prognostic genes in kidney renal clear cell carcinoma by RNA-seq data analysis. Mol Med Rep. (2017) 15:1661-67. doi: 10.3892/mmr.2017.6194

21. Su Q, Ding Q, Zhang Z, Yang Z, Qiu Y, Li X, et al. Identification of genes associated with the metastasis of Pheochromocytoma/Paraganglioma based on weighted gene coexpression network analysis. Biomed Res Int. (2020) 2020:3876834. doi: 10.1155/2020/3876834
22. Langfelder $\mathrm{P}$, Horvath S. WGCNA: an $\mathrm{R}$ package for weighted correlation network analysis. BMC Bioinform. (2008) 9:559. doi: 10.1186/1471-2105-9-559

23. Pei G, Chen L, Zhang W. WGCNA application to proteomic and metabolomic data analysis. Methods Enzymol. (2017) 585:135-58. doi: 10.1016/bs.mie.2016.09.016

24. Li S, Liu X, Liu T, Meng X, Yin X, Fang C, et al. Identification of biomarkers correlated with the TNM staging and overall survival of patients with bladder cancer. Front Physiol. (2017) 8:947. doi: 10.3389/fphys.2017.00947

25. Zhai X, Xue Q, Liu Q, Guo Y, Chen Z. Colon cancer recurrenceassociated genes revealed by WGCNA coexpression network analysis. Mol Med Rep. (2017) 16:6499-505. doi: 10.3892/mmr.2017.7412

26. Zhang $\mathrm{W}, \mathrm{Xu} \mathrm{Y,} \mathrm{Zhang} \mathrm{L,} \mathrm{Wang} \mathrm{S,} \mathrm{Yin} \mathrm{B,} \mathrm{Zhao} \mathrm{S,} \mathrm{et} \mathrm{al.} \mathrm{Synergistic}$ effects of TGF $\beta 2$, WNT9a, and FGFR4 signals attenuate satellite cell differentiation during skeletal muscle development. Aging Cell. (2018) 17:e12788. doi: 10.1111/acel.12788

27. Yu G, Wang LG, Han Y, He QY. clusterProfiler: an $\mathrm{R}$ package for comparing biological themes among gene clusters. Omics. (2012) 16:284-7. doi: 10.1089/omi.2011.0118

28. Bhattacharya S, Andorf S, Gomes L, Dunn P, Schaefer H, Pontius J, et al. ImmPort: disseminating data to the public for the future of immunology. Immunol Res. (2014) 58:234-9. doi: 10.1007/s12026-014-8516-1

29. Leek JT, Johnson WE, Parker HS, Jaffe AE, Storey JD. The sva package for removing batch effects and other unwanted variation in high-throughput experiments. Bioinformatics. (2012) 28:882-3. doi: 10.1093/bioinformatics/bts034

30. Goeman JJ. L1 penalized estimation in the Cox proportional hazards model. Biom J. (2010) 52:70-84. doi: 10.1002/bimj.200900028

31. Friedman J, Hastie T, Tibshirani R. Regularization paths for generalized linear models via coordinate descent. J Stat Softw. (2010) 33:1-22. doi: $10.18637 /$ jss.v033.i01

32. Yoshihara K, Shahmoradgoli M, Martinez E, Vegesna R, Kim H, TorresGarcia W, et al. Inferring tumour purity and stromal and immune cell admixture from expression data. Nat Commun. (2013) 4:2612. doi: $10.1038 /$ ncomms 3612

33. Newman AM, Liu CL, Green MR. Robust enumeration of cell subsets from tissue expression profiles. Nat Methods. (2015) 12:453-7. doi: 10.1038/nmeth.3337

34. Schreiber RD, Old LJ, Smyth MJ. Cancer immunoediting: integrating immunity's roles in cancer suppression and promotion. Science. (2011) 331:1565-70. doi: 10.1126/science. 1203486

35. Chowell D, Morris LGT, Grigg CM, Weber JK, Samstein RM, Makarov $\mathrm{V}$, et al. Patient HLA class I genotype influences cancer response to checkpoint blockade immunotherapy. Science. (2018) 359:582-7. doi: $10.1126 /$ science.aao 4572

36. Pardoll DM. The blockade of immune checkpoints in cancer immunotherapy. Nat Rev Cancer. (2012) 12:252-64. doi: 10.1038/nrc3239

37. Mayakonda A, Lin DC, Assenov Y, Plass C, Koeffler HP. Maftools: efficient and comprehensive analysis of somatic variants in cancer. Genome Res. (2018) 28:1747-56. doi: 10.1101/gr.239244.118

38. Robinson DR, Wu YM, Lonigro RJ, Vats P, Cobain E, Everett J, et al. Integrative clinical genomics of metastatic cancer. Nature. (2017) 548:297303. doi: $10.1038 /$ nature 23306

39. Subramanian A, Tamayo P, Mootha VK, Mukherjee S, Ebert BL, Gillette MA, et al. Gene set enrichment analysis: a knowledge-based approach for interpreting genome-wide expression profiles. Proc Natl Acad Sci USA. (2005) 102:15545-50. doi: 10.1073/pnas.0506580102

40. Zhou W, Laird PW, Shen H. Comprehensive characterization, annotation and innovative use of Infinium DNA methylation BeadChip probes. Nucleic Acids Res. (2017) 45:e22. doi: 10.1093/nar/gkw967

41. Conforti F, Pala L, Bagnardi V, de Pas T, Martinetti M, Viale G, et al. Cancer immunotherapy efficacy and patients' sex: a systematic review and meta-analysis. Lancet Oncol. (2018) 19:737-46. doi: 10.1016/S1470-2045(18)30261-4

42. Tawbi HA, Burgess M, Bolejack V, van Tine BA, Schuetze SM, Hu J, et al. Pembrolizumab in advanced soft-tissue sarcoma and bone sarcoma (SARC028): a multicentre, two-cohort, single-arm, open-label, phase 2 trial. Lancet Oncol. (2017)18:1493-501. doi: 10.1016/S1470-2045(17)30624-1 
43. Toulmonde M, Penel N, Adam J, Chevreau C, Blay JY, Le Cesne A, et al. Use of PD-1 Targeting. Macrophage infiltration, and ido pathway activation in sarcomas: a phase2 clinical trial. JAMA Oncol. (2018) 4:93-7. doi: 10.1001/jamaoncol.2017.1617

44. Danaher P, Warren S, Lu R, Samayoa J, Sullivan A, Pekker I, et al. Pan-cancer adaptive immune resistance as defined by the Tumor Inflammation Signature (TIS): results from The Cancer Genome Atlas (TCGA). J Immunother Cancer. (2018) 6:63. doi: 10.1186/s40425-018-0367-1

45. Turan T, Kannan D, Patel M, Matthew Barnes J, Tanlimco SG, Lu R, et al. Immune oncology, immune responsiveness and the theory of everything. $J$ Immunother Cancer. (2018) 6:50. doi: 10.1186/s40425-018-0355-5

46. Liu J, Nie S, Wu Z, Jiang Y, Wan Y, Li S, et al. Exploration of a novel prognostic risk signatures and immune checkpoint molecules in endometrial carcinoma microenvironment. Genomics. (2020) 112:3117-34. doi: 10.21203/rs.3.rs-18621/v1

47. Yu X, Wang H, Li X, Guo C, Yuan F, Fisher PB, et al. Activation of the MDA-5-IPS-1 viral sensing pathway induces cancer cell death and Type I IFN-dependent antitumor immunity. Cancer Res. (2016) 76:2166-76. doi: 10.1158/0008-5472.CAN-15-2142

48. Yamazaki T, Aoki Y. Cathepsin G enhances human natural killer cytotoxicity. Immunology. (1998) 93:115-21. doi: 10.1046/j.1365-2567.1998.00397.x

49. Yang S, Ji Q, Chang B, Wang Y, Zhu Y, Li D, et al. STC2 promotes head and neck squamous cell carcinoma metastasis through modulating the PI3K/AKT/Snail signaling. Oncotarget. (2017) 8:5976-91. doi: 10.18632/oncotarget. 13355

50. Wu F, Li TY, Su SC, Yu JS, Zhang HL, Tan GQ, et al. STC2 as a novel mediator for Mus81-dependent proliferation and survival in hepatocellular carcinoma. Cancer Lett. (2017) 388:177-86. doi: 10.1016/j.canlet.2016.11.039

51. Na SS, Aldonza MB, Sung HJ, Kim YI, Son YS, Cho S, et al. Stanniocalcin2 (STC2): a potential lung cancer biomarker promotes lung cancer metastasis and progression. Biochim Biophys Acta. (2015) 1854:668-76. doi: 10.1016/j.bbapap.2014.11.002

52. Wang B, Li X, Zhao G, Yan H, Dong $\mathrm{P}$, Watari $\mathrm{H}$, et al. miR203 inhibits ovarian tumor metastasis by targeting BIRC5 and attenuating the TGF $\beta$ pathway. J Exp Clin Cancer Res. (2018) 37:235. doi: 10.1186/s13046-018-0906-0

53. Wang T, Huang C, Lopez-Coral A, Slentz-Kesler KA, Xiao M, Wherry EJ, et al. K12/SECTM1, an interferon-gamma regulated molecule, synergizes with CD28 to costimulate human T cell proliferation. J Leukoc Biol. (2012) 91:449-59. doi: 10.1189/jlb.1011498

54. Andre P, Denis C, Soulas C, Bourbon-Caillet C, Lopez J, Arnoux T, et al. Anti-NKG2A mAb Is a checkpoint inhibitor that promotes anti-tumor immunity by unleashing both T and NK cells. Cell. (2018) 175:1731-43.e13. doi: 10.1016/j.cell.2018.10.014

55. Bigley $\mathrm{AB}$, Simpson RJ. NK cells and exercise: implications for cancer immunotherapy and survivorship. Discov Med. (2015) 19:433-45.

56. Li X, Cai Y. Better prognostic determination and feature characterization of cutaneous melanoma through integrative genomic analysis. Aging. (2019) 11:5081-107. doi: 10.18632/aging.102099
57. Woo SR, Fuertes MB, Corrales L, Spranger S, Furdyna MJ, Leung MY, et al. STING-dependent cytosolic DNA sensing mediates innate immune recognition of immunogenic tumors. Immunity. (2014) 41:830-42. doi: 10.1016/j.immuni.2014.10.017

58. Guedan S, Posey AD Jr, Shaw C, Wing A, Da T, Patel PR, et al. Enhancing CAR $\mathrm{T}$ cell persistence through ICOS and 4-1BB costimulation. JCI Insight. (2018) 3:e96976. doi: 10.1172/jci.insight.96976

59. Stephan MT, Ponomarev V, Brentjens RJ, Chang AH, Dobrenkov KV, Heller G, et al. T cell-encoded CD80 and 4-1BBL induce auto- and transcostimulation, resulting in potent tumor rejection. Nat Med. (2007) 13:1440-9. doi: 10.1038/nm1676

60. Xiao Y, Ma D, Zhao S, Suo C, Shi J, Xue MZ, et al. Multi-omics profiling reveals distinct microenvironment characterization and suggests immune escape mechanisms of triple-negative breast cancer. Clin Cancer Res. (2019) 25:5002-14. doi: 10.1158/1078-0432.CCR-18-3524

61. Liu Y, Cao X. Immunosuppressive cells in tumor immune escape and metastasis. J Mol Med. (2016) 94:509-22. doi: 10.1007/s00109-015-1376-X

62. Chen G, Huang AC, Zhang W, Zhang G, Wu M, Xu W, et al. Exosomal PD-L1 contributes to immunosuppression and is associated with anti-PD-1 response. Nature. (2018) 560:382-6. doi: 10.1038/s41586-018-0392-8

63. Conforti F, Pala L, Bagnardi V, Viale G, de Pas T, Pagan E, et al. Sex-based heterogeneity in response to lung cancer immunotherapy: a systematic review and meta-analysis. J Natl Cancer Inst. (2019) 111:772-81. doi: 10.1093/jnci/djz094

64. Wallis CJD, Butaney M, Satkunasivam R, Freedland SJ, Patel SP, Hamid O, et al. Association of patient sex with efficacy of immune checkpoint inhibitors and overall survival in advanced cancers: a systematic review and metaanalysis. JAMA Oncol. (2019) 5:529-36. doi: 10.1001/jamaoncol.2018.5904

65. Mutation Burden Predicts Anti-PD-1 Response. Cancer Discov. (2018) 8:258. doi: 10.1158/2159-8290.CD-NB2018-005

66. Cancer Genome Atlas Research Network. Comprehensive and integrated genomic characterization of adult soft tissue sarcomas. Cell. (2017) 171:95065.e28. doi: 10.1016/j.cell.2017.10.014

67. MHC expression predicts checkpoint blockade response. Cancer Discov. (2018) 8:1052. doi: 10.1158/2159-8290.CD-NB2018-104

68. Savic Prince S, Bubendorf L. Predictive potential and need for standardization of PD-L1 immunohistochemistry. Virchows Arch. (2019) 474:475-84. doi: $10.1007 / \mathrm{s} 00428-018-2445-7$

Conflict of Interest: The authors declare that the research was conducted in the absence of any commercial or financial relationships that could be construed as a potential conflict of interest.

Copyright (c) $2020 \mathrm{Gu}$, Lin, Zhang, Yang, Zhong and Wei. This is an open-access article distributed under the terms of the Creative Commons Attribution License (CC $B Y)$. The use, distribution or reproduction in other forums is permitted, provided the original author(s) and the copyright owner(s) are credited and that the original publication in this journal is cited, in accordance with accepted academic practice. No use, distribution or reproduction is permitted which does not comply with these terms. 